\title{
Micro Model of Carbon Fiber/Cyanate Ester Composites and Analysis of Machining Damage Mechanism
}

\author{
Haitao Liu' ${ }^{1}$ Jie Lin ${ }^{1}$, Yazhou Sun ${ }^{1 *}$ and Jinyang Zhang ${ }^{2}$
}

\begin{abstract}
Machining damage occurs on the surface of carbon fiber reinforced polymer (CFRP) composites during processing. In the current simulation model of CFRP, the initial defects on the carbon fiber and the periodic random distribution of the reinforcement phase in the matrix are not considered in detail, which makes the characteristics of the cutting model significantly different from the actual processing conditions. In this paper, a novel three-phase model of carbon fiber/cyanate ester composites is proposed to simulate the machining damage of the composites. The periodic random distribution of the carbon fiber reinforced phase in the matrix was realized using a double perturbation algorithm. To achieve the stochastic distribution of the strength of a single carbon fiber, a novel method that combines the Weibull intensity distribution theory with the Monte Carlo method is presented. The mechanical properties of the cyanate matrix were characterized by fitting the stress-strain curves, and the cohesive zone model was employed to simulate the interface. Based on the model, the machining damage mechanism of the composites was revealed using finite element simulations and by conducting a theoretical analysis. Furthermore, the milling surfaces of the composites were observed using a scanning electron microscope, to verify the accuracy of the simulation results. In this study, the simulations and theoretical analysis of the carbon fiber/cyanate ester composite processing were carried out based on a novel three-phase model, which revealed the material failure and machining damage mechanism more accurately.
\end{abstract}

Keywords: Carbon fiber reinforced polymer composites, Micro simulation model, Machining damage mechanism, Milling and observation experiment, Theoretical analysis

\section{Introduction}

Carbon fiber reinforced polymer (CFRP) composites have been extensively used in various industries, including aerospace, transportation, and medical. This is because of their excellent mechanical and physical properties [1-4]. However, due to the significant difference between the mechanical properties of carbon fibers and the matrix, the machining of CFRP composites is relatively difficult to achieve $[5,6]$. During the mechanical machining process of CFRP composites, various defects such as delamination, tearing, burring, and heat damage

\footnotetext{
*Correspondence: sunyzhit@163.com

${ }^{1}$ Center for Precision Engineering, School of Mechanical Engineering,

Harbin Institute of Technology, Harbin 150001, China

Full list of author information is available at the end of the article
}

could be easily formed on the machined surface, which significantly affects the performance of the machined surface [7-10]. Therefore, to obtain a higher surface quality, it is necessary to determine the machining damage mechanism of CFRP composites.

Compared with the machining experiments of composite materials, which are expensive, time-consuming, and harmful to health; numerical simulation, which can realize the machining process under various complex conditions, is an important method for the evaluation of the machining mechanism of composite materials. In addition, the related parameters can be easily changed. However, it is difficult to establish a simulation model due to the anisotropy of the composite [11, 12]. At present, the numerical simulation model for the CFRP composites 
can be divided into two categories: macro and micro models.

In the past few decades, scholars have directed significant research effort toward the construction of macro models. Mkaddem et al. developed a model that considers composite materials as homogeneous materials, and then analyzed the main factors that influence the surface damage [13]. Mahdi et al. conducted a simulation of the two-dimensional orthogonal cutting of composite materials using a macro model, and then analyzed the influence of the tool parameters on the cutting force and material surface damage [14, 15]. A macro indentation test model was presented, and the CFRP composite laminates were evaluated further by Sun et al., to reveal the interaction between matrix cracks and delamination [16, 17]. Rao et al. [18] presented a three-dimensional model using the finite element method (FEM), and then evaluated the effects of the fiber orientation and tool parameters on the surface damage mechanism of the composites. The chip formation mechanism and sub-surface damage of unidirectional carbon fiber reinforced composites were analyzed using Zenia, which considers the plastic deformation process of the composites [19]. In the current macro models, the material is typically regarded as an equivalent homogeneous material, and the interaction between the fibers and matrix is neglected. Therefore, it is difficult to analyze the surface damage of composites accurately.

When compared with the macro models, the microscopic models can be applied to different components, in which the machining damage of the composites can be observed and analyzed effectively. The damage mechanism can therefore be evaluated in detail. Hence, in recent years, significant research attention has been directed toward the establishment of an accurate micro model of CFRP composites [20]. Based on previous studies, Dikshit et al. [21] proposed a microscopic geometry model of composites, which can control the microstructural parameters of the material, in addition to the fiber orientation. Rentsch et al. [22] established a finite element micro model of carbon fiber reinforced composites, and then analyzed the damage mechanism of composites. However, the composites were only considered as twophase materials, which included the fiber and matrix, thus yielding inaccurate simulation results. A threephase microscopic model for composites was successfully developed by Abena et al. [23], in which a finite element with minimal thickness was used to model the interface. The influence of the tool parameters on the interphase damage was discussed. Wang et al. [24] set up the macro and micro models separately for the composites; however, the fiber-reinforced phase was simplified as a homogeneous brittle material. The removal processes of materials at the macro and micro scales were compared, and the results revealed that the degree of surface damage of the fiber, matrix, and interface were not the same under different conditions. Similarly, Yang et al. [25] developed a multi-scale finite element model of composites to evaluate the damage mechanism of materials under low velocity impact, which indicated that the matrix cracks initially occurred, and the delamination and fiber fracture were then initiated. Moreover, Zhu et al. $[26,27]$ proposed an inverse strategy (based on the Kriging metamodel) to simulate the three-phase finite element model of fiber-reinforced composites.

In summary, the current research on CFRP composites has been successful. However, there are several drawbacks. The initial defects of the carbon fiber and the periodic random distribution of the reinforced phase in the matrix are not considered. Consequently, the characteristics of the cutting model for the composites should be improved. Further, because of the lack of a relevant theoretical analysis, the studies on the damage mechanism of composites are limited to the exploration of the effect of the cutting parameters on the surface damage. Therefore, they cannot reveal the machining damage mechanism of the composites. Moreover, few reports on the coupling effect between the initial defect and the machining damage were found.

In this study, a novel three-phase FEM model that consists of carbon fiber, cyanate ester, and interface phase was developed based on ABAQUS, which considers the initial defects of the carbon fiber reinforced phase and the periodic random distribution of fiber in the matrix. In addition, the underlying cutting damage mechanism of composites during the machining process was revealed using FEM simulations and a theoretical analysis. The SEM observations of the milling surfaces of the carbon fiber/cyanate ester composites milling surface were carried out to validate the accuracy of the simulation results.

\section{Model Development}

\subsection{Geometry Model of Carbon Fiber/Cyanate Ester Composites}

Carbon fiber/cyanate composite is a typical type of CFRP composite. In the cyanate matrix, the carbon fibers are distributed parallel to each other. The establishment of the geometric model of CFRP composites can be initiated with the design of the cross-section. The composites can be simplified as a unit volume, which is a certain number of fibers within the cross-section. In addition, the center of each fiber is randomly distributed in the cross-section. The actual cross-section of the unidirectional CFRP composites observed by SEM is presented in Figure 1.

In the model, a double perturbation algorithm is used to realize the random distribution of the fibers in the 


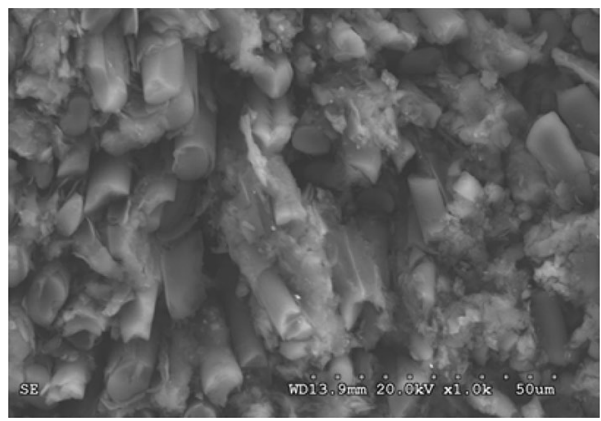

Figure 1 Cross-section of unidirectional CFRP composites

matrix. The first step is the global perturbation of the center coordinates of the fiber, and the displacement is added by interlacing. The red and blue fibers in Figure 2(a) complete the right and left displacement disturbance $\Delta x$, respectively; and the red and blue fibers in Figure 2(b) complete the up and down displacement disturbance $\Delta y$, respectively.

The second step is the local perturbation of the center coordinates of the fiber, as shown in Figure 3. Three neighboring carbon fibers were assumed, labelled as fibers 1,2 , and 3 . The coordinates of fiber 1 are $\left(x_{0}, y_{0}\right)$. In addition, $R$ is the smallest distance between fiber 1 and the adjacent fiber, which indicates the range of the local perturbation of the single fiber. The center of the fiber
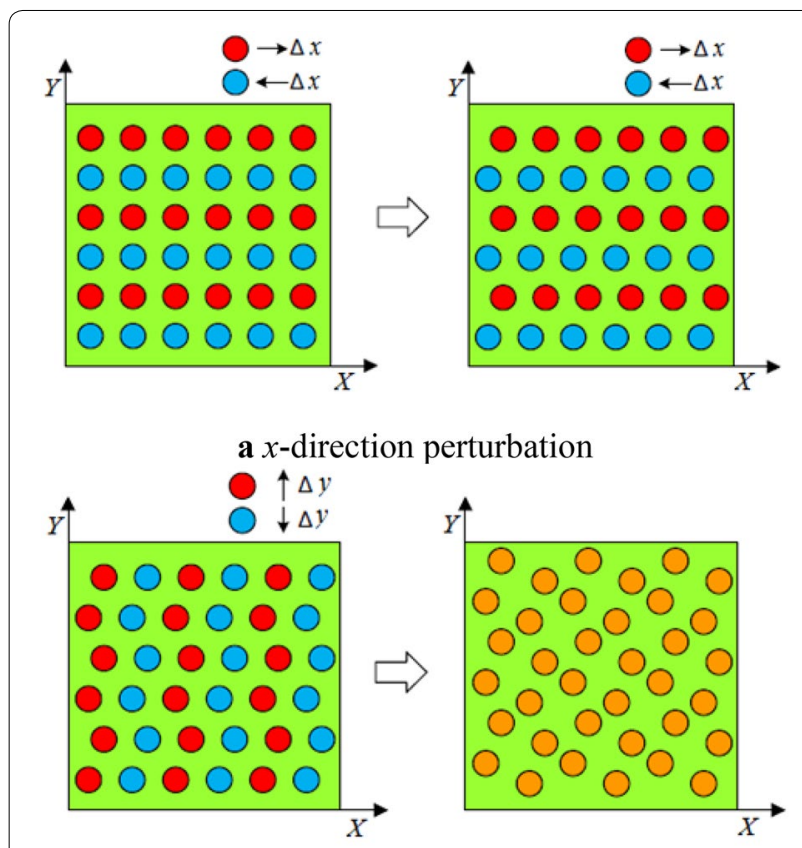

b $y$-direction perturbation

Figure 2 Global perturbation of fiber coordinates
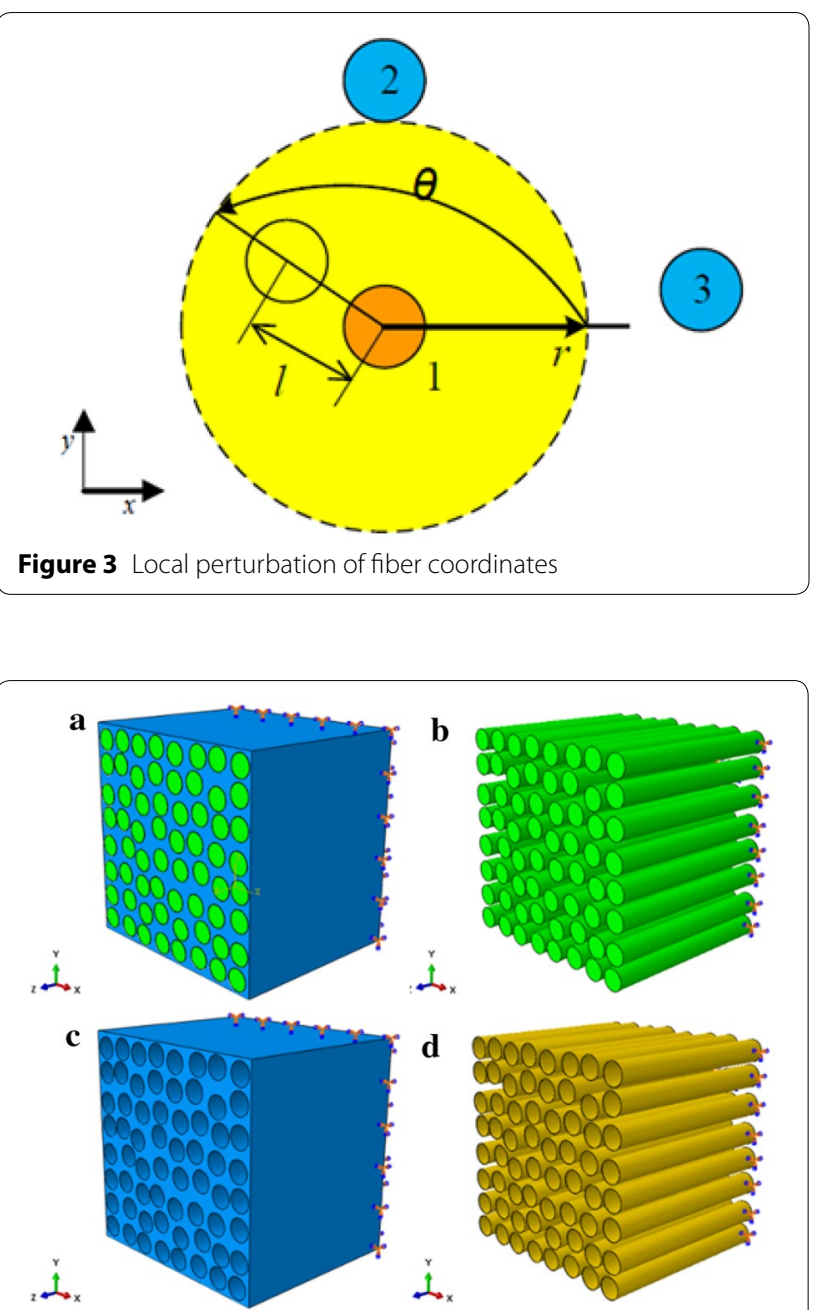

Figure 4 Geometry model of carbon fiber/cyanate ester composites

was considered as the origin, and the coordinate system was established. If the angle between the fiber perturbation direction and $x$ axis is $\theta_{i}$, the distance of the fiber disturbance is then $l_{i}=\alpha_{i} R$; where $\theta_{i}$ and $\alpha_{i}$ are the random values generated in the random number generator with the python statement. Moreover, $\theta_{i}$ is in the range of $(0,2 \pi)$, and $\alpha_{i}$ is in the range of $(0,1)$. After the local perturbation, the new center coordinate of the fiber is $\left(x_{0}\right.$ $\left.+l_{i} \cos \theta_{i}, y_{0}+l_{i} \sin \theta_{i}\right)$. Finally, a micro-geometry model with a periodic random distribution of these fibers in the matrix was obtained, as shown in Figure 4.

\subsection{Material Model of Carbon Fiber/Cyanate Ester Composites}

\subsubsection{Material Model of Carbon Fiber Reinforced Phase}

Carbon fiber, as a typical brittle material, generally has randomly-distributed defects that occur during its 
preparation process (such as cracks and holes), which significantly affect its mechanical strength. Tomonaga Okabe and Nobuo Takeda proposed a method that combines the Weibull intensity distribution formula with the Monte Carlo method, for the simulation of the carbon fibers with randomly-distributed strengths, as shown in Eq. (1). Moreover, the accuracy of this method was experimentally verified [28].

$$
\sigma_{\mathrm{s}}=\left(\frac{L_{0}}{\delta} \ln \frac{1}{1-R}\right)^{1 / m} \sigma_{0}-E_{f} \varepsilon_{f}^{\text {residual }},
$$

where $R$ is a random value in the range of $(0,1), \delta$ is the unit length $(\mathrm{m}), \varepsilon_{f}^{\text {residual }}$ is the residual strain due to the temperature change during the machining process, $m$ is the Weibull shape parameter, $\sigma_{0}$ is the Weibull proportional parameter $(\mathrm{MPa})$, and $L_{0}$ is the characteristic length of the carbon fiber ( $\mathrm{m})$.

In this study, the abovementioned method was applied to establish the carbon fiber model. Each carbon fiber was divided into several small segments, and a tensile strength value was randomly assigned to each segment according to Eq. (1), to realize the random distribution of the initial defects. The parameters used in the formula are presented in Table 1.

\subsubsection{Material Model of Cyanate Ester Matrix and Interface}

The constitutive model of the cyanate ester material is based on the stress-strain curve in Ref. [30]. The tensile strength of the cyanate ester matrix is $69.9 \mathrm{MPa}$, the elastic modulus is $2037 \mathrm{MPa}$, and the elongation is $5.5 \%$. The Mises criterion is used to determine whether the material reaches the yield point; and when the material is in the plastic deformation stage, the failure criterion is used to determine whether the material has reached the failure stage.

The thickness of the interface between the fiber and matrix is very small; thus, the conventional finite element cannot be used to simulate the interface. In this study, the cohesive zone model with the

Table 1 Carbon fiber performance parameters [29]

\begin{tabular}{ll}
\hline Parameters of carbon fiber & Values \\
\hline Elastic modulus $(\mathrm{GPa})$ & 230 \\
Poisson's ratio $\mathrm{V}$ & 0.3 \\
Weibull shape parameter $(\mathrm{m})$ & 10.39 \\
Weibull proportional parameter $\sigma_{0}$ & 2800 \\
The characteristic length $L_{0}(\mathrm{~mm})$ & 1 \\
Diameter $D(\mu \mathrm{m})$ & 7 \\
\hline
\end{tabular}

traction-separation criterion was employed to solve this problem. Further, the maximum nominal stress criterion was used to determine the initial damage point of the material, and linear attenuation based on energy was adopted as the softening criterion.

\subsection{Micro Cutting Model of Carbon Fiber/Cyanate Ester Composites}

A finite element model was established to simulate the micro-cutting process of carbon fiber/cyanate ester composites, as shown in Figure 5. The cutting model mainly consists of carbon fiber/cyanate ester composites and the micro tool; in which the carbon fiber reinforced phase and matrix are meshed using C3D8R units, whereas the interfacial phase is meshed by COH3D8 units.

The tie constraint was used for the carbon fiber reinforced phase, matrix, and interfacial phase. Moreover, the carbon fibers and matrix were set as the main face, whereas the interfacial phase was considered as the main surface. The micro tool was simplified to an ideal rigid body, and the motion of the micro tool was controlled to carry out the cutting of the carbon fiber/cyanate ester composites. The rake angle, relief angle, and cutting edge blunt round radius of the micro tool were $10^{\circ}, 25^{\circ}$, and $2 \mu \mathrm{m}$, respectively. In addition, the bottom surface of the carbon fiber/cyanate ester composites was constrained by the fixed boundary condition, whereas both sides along the cutting direction were constrained by the symmetric boundary condition.

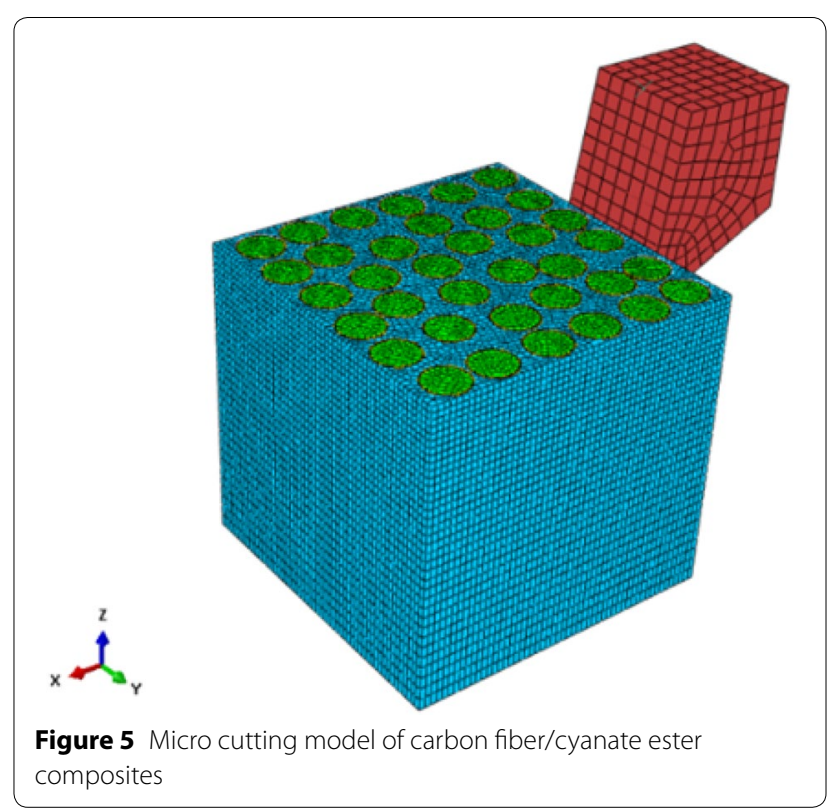




\section{Results and Discussion}

\subsection{Model Verification}

To verify the material model for carbon fiber/cyanate ester composites, the typical surface damage obtained from conducting experiments and simulations were compared. Figure 6 presents the SEM and simulation snapshots of the hole defects, as induced by the fracture of a local single carbon fiber. As displayed, the simulation results were approximately consistent with the experimental results. The formation of the hole defect is due to the inherent defect of carbon fiber. When the carbon fiber/cyanate ester composites are cut off by the tool, the carbon fiber can be easily fractured at the damaged point, thus inducing the formation of the hole defect. Under the extrusion interactions of the tool, the carbon fibers bend along the cutting direction, which leads to the fracture of the matrix material and other carbon fibers. Moreover, the stress concentration regions are formed on the machined surface of the CFRP composites, which leads the extension of cracks.

During the cutting process of carbon fiber, the fibers tend to bend under the extrusion of tool. However, due to the difference in the position and cutting force of each
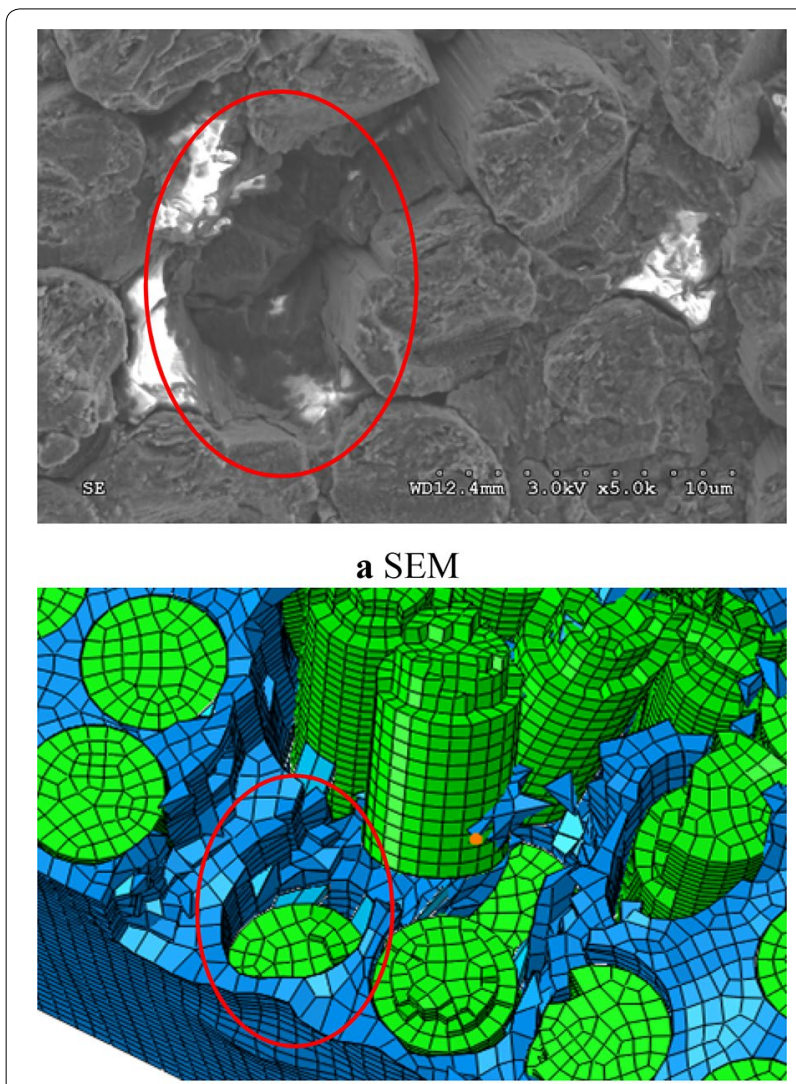

b Simulation

Figure 6 Hole defects due to the fracture of local single carbon fiber fiber, the gaps between the carbon fibers and matrix are easily formed, thereby resulting in the formation of surface cracks. Figure 7 illustrates the surface cracks induced by the elastic bending deformation of carbon fiber.

In addition, air holes or incomplete adhesion between the carbon fibers and the surrounding matrix are easily formed in the manufacturing process of carbon fiber/ cyanate ester composites, thus decreasing the interface bonding strength. During the cutting process of CFRP composites, cracks are easily and preferentially formed. Moreover, the stress concentration areas formed during the cutting process tend to promote the extension of the cracks further, and long cracks with large depths are easily formed on the machined surface. When the CFRP composites are cut by the micro tool, the material in the cracks is removed in the form of adhesive shedding, and surface pits are generated, as shown in Figure 8.

Finally, Figure 9 presents the SEM and simulation snapshots of the thin elongated cracks induced by
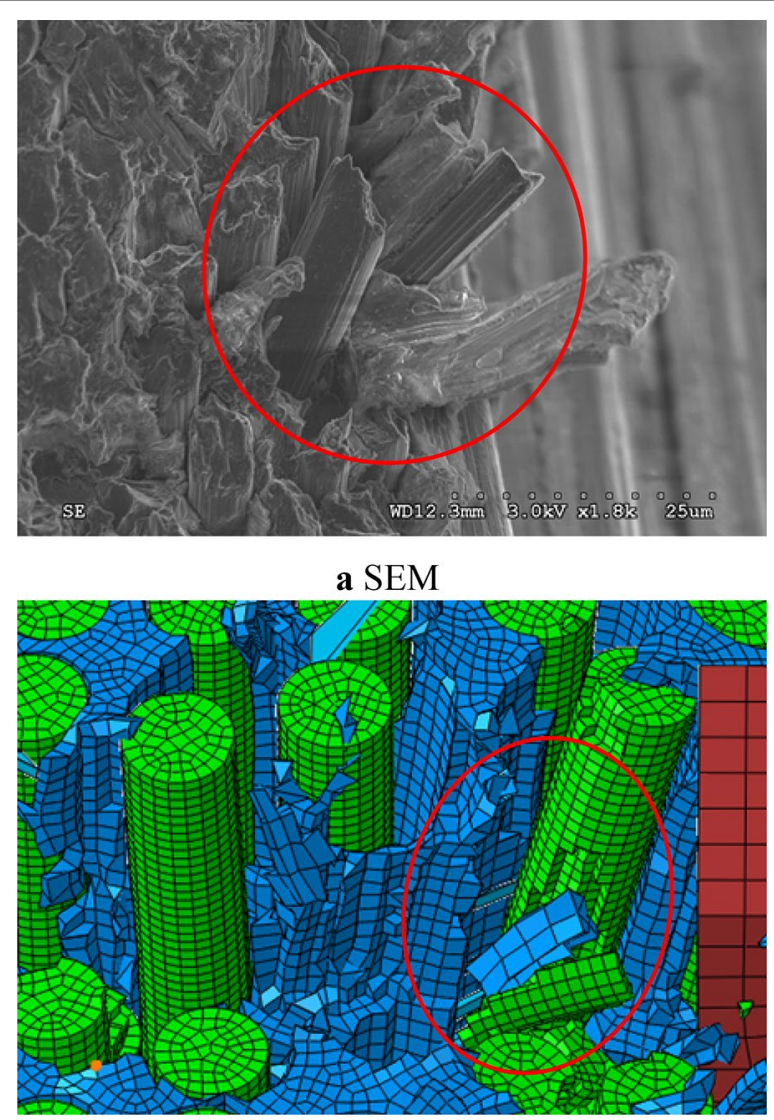

b Simulation

Figure 7 Surface cracks due to elastic bending deformation of carbon fiber 


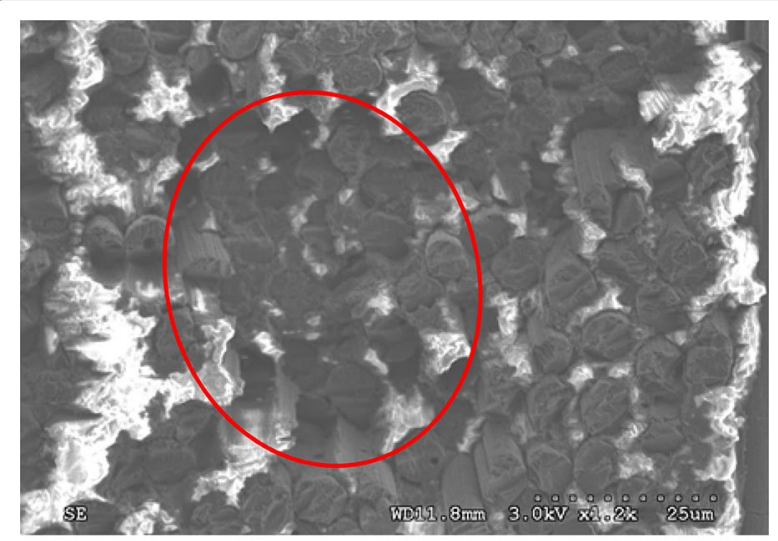

a SEM

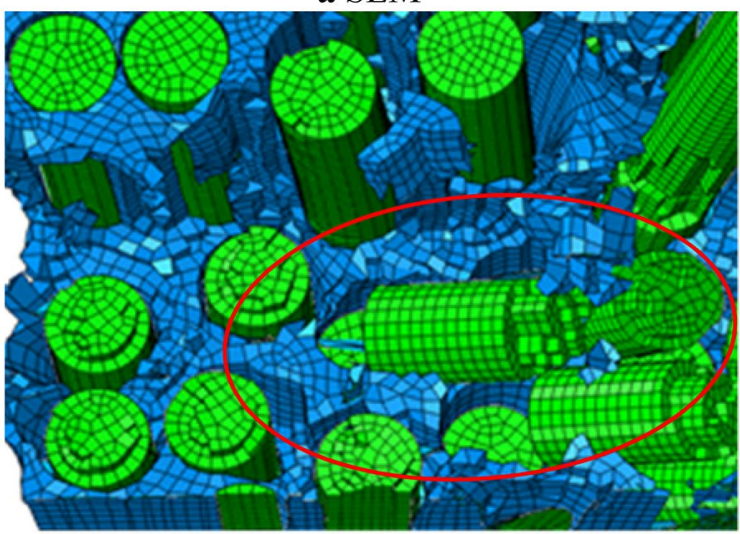

b Simulation

Figure 8 Air holes or incomplete adhesion between carbon fibers and surrounding matrix

the shedding carbon fibers, which typically occurs when the cutting is carried out at a fiber orientation of approximately $0^{\circ}$. Under the extrusion of the tool, cracks are easily formed in the carbon fibers. With the application of the micro tool, the bonding strength between carbon fibers and matrix gradually decreases. When the shear stress between the carbon fibers and matrix is higher than the bonding strength, the fractured carbon fibers and matrix materials are removed. Thin pores are then easily formed on the processed surface.

\subsection{Simulation Results for Machining Damage of Carbon Fiber/Cyanate Ester Composites}

In this section, an analysis of the machining-induced damages on the carbon fiber/cyanate ester composites
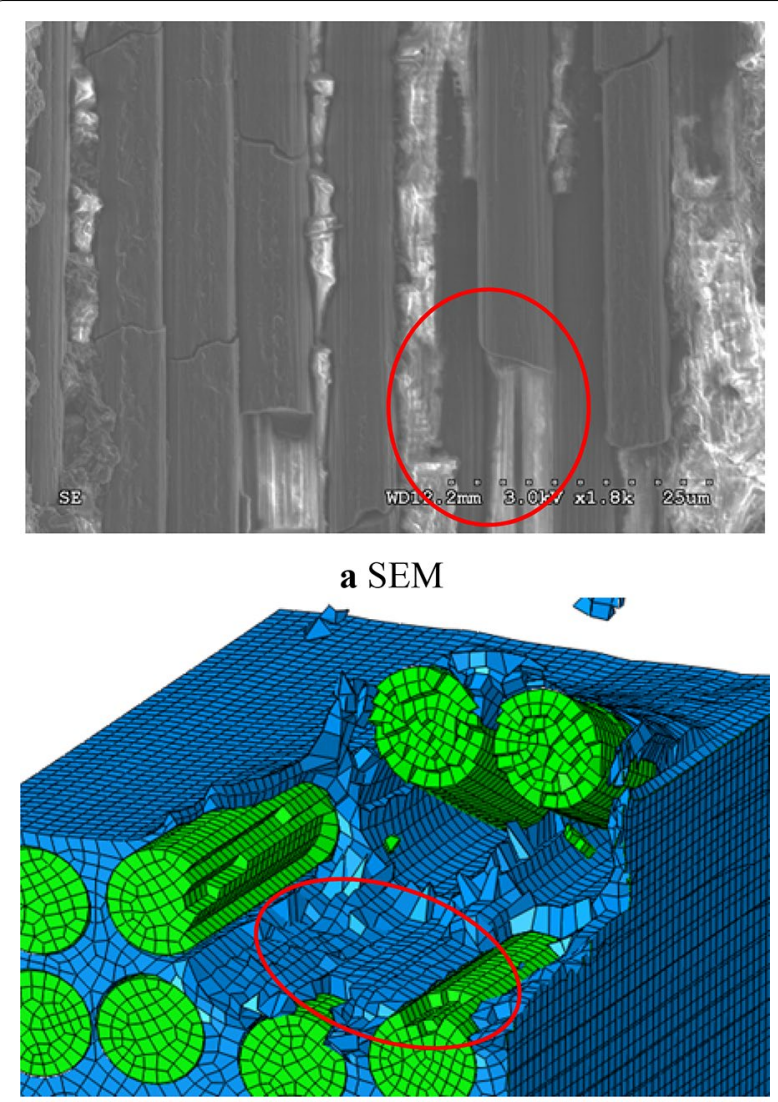

b Simulation

Figure 9 Thin elongated cracks induced by shedding carbon fibers

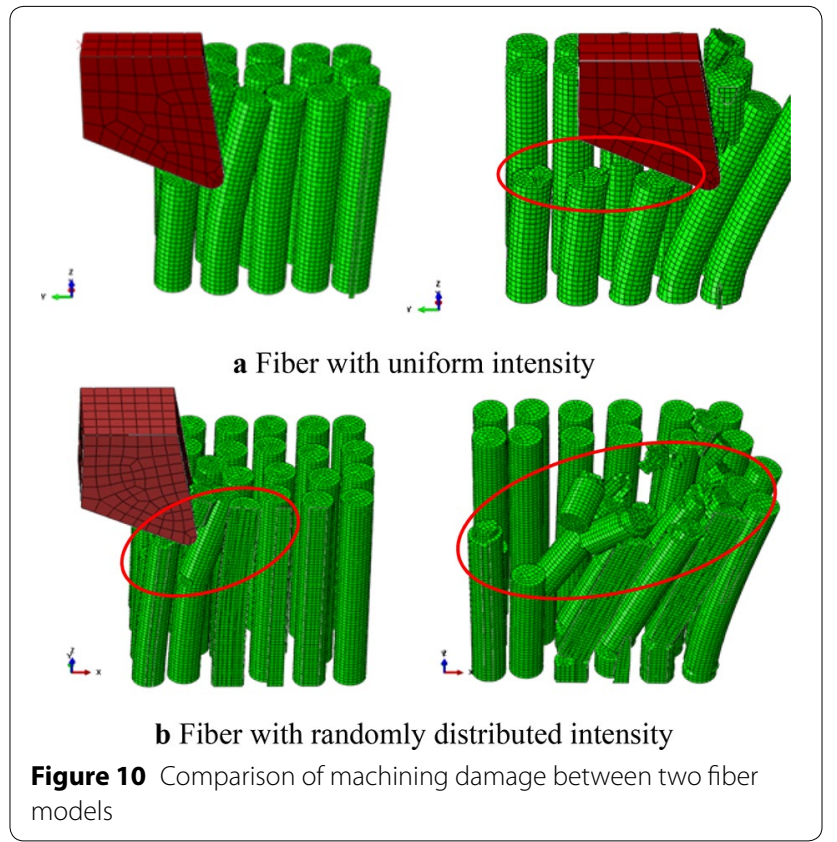


were analyzed with respect to the carbon fiber reinforced phase, matrix, and interface phase.

\subsubsection{Machining Damage of Carbon Fiber Reinforced Phase}

At the reinforced phase in CFRP composites, the strength of the carbon fibers and elastic modulus are significantly higher than those of the cyanate ester matrix. Hence, during the cutting process, different failures tend to occur in the carbon fibers and matrix, which are mainly dependent on the strength distribution of carbon fibers and the cutting conditions. To indicate the effect of the strength distribution on the cutting process of the carbon fibers clearly, the carbon fibers with uniform and Weibull strength distributions were employed. Figure 10 presents the removal processes of carbon fibers with different strength distributions. As shown, for those with uniform mechanical strength distributions, carbon fibers were cut-out at the positions with similar depths, and the fractured cross-sections were neat. In contrast, for those carbon fibers with random strength distributions, they were fractured at different depths under the extrusion of the micro tool. This is due to the intrinsic defects of carbon fibers. It is known that the strength of the carbon fibers at the defects tends to be lower; thus, the fibers are easily fractured in the cutting process. Therefore, to describe the material removal process of carbon fibers/cyanate ester composites accurately, the Weibull strength distribution could be preferentially considered.

\subsubsection{Machining Damage of Cyanate Ester Matrix}

In the carbon fiber/cyanate ester composites, the mechanical strength of the carbon fibers is approximately higher than that of the cyanate ester matrix by a factor of 10 . In the cutting process, the matrix is preferentially destroyed and removed, thus leading to the further formation of cracks in the matrix. It can be clearly seen in Figure 11 that several micro cracks were formed in the machined cyanate ester matrix. Moreover, regions of the matrix were clearly crushed.

According to the basis theory of fracture mechanics, the cracks could be divided into the "open" (I), "sliding" (II), and "tear" (III) categories, as shown in Figure 12. In this section, an analysis of the formation and evolution

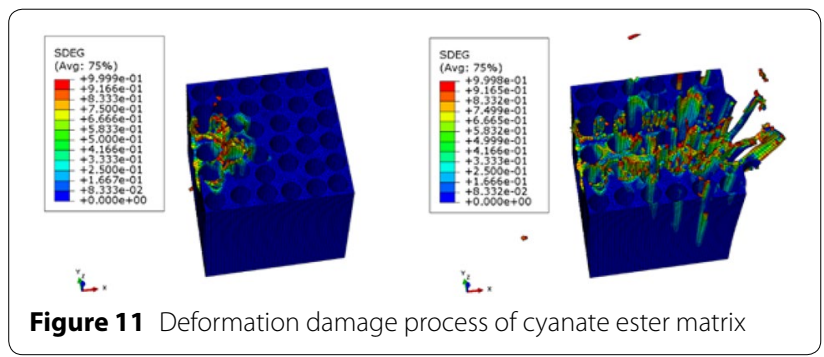

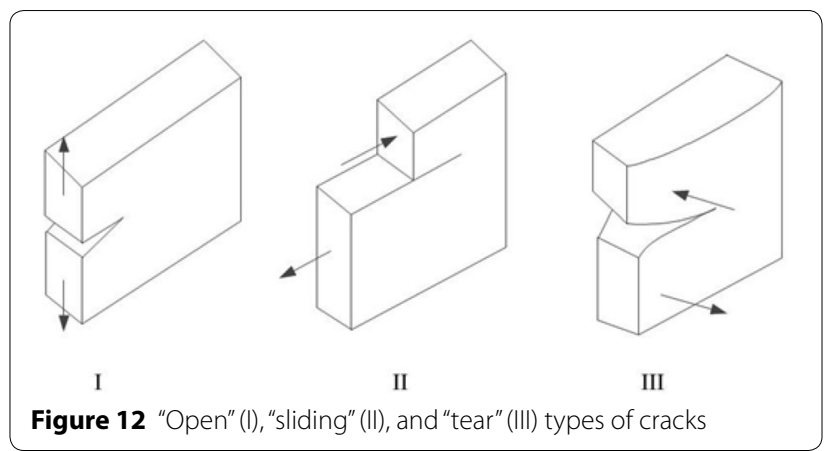

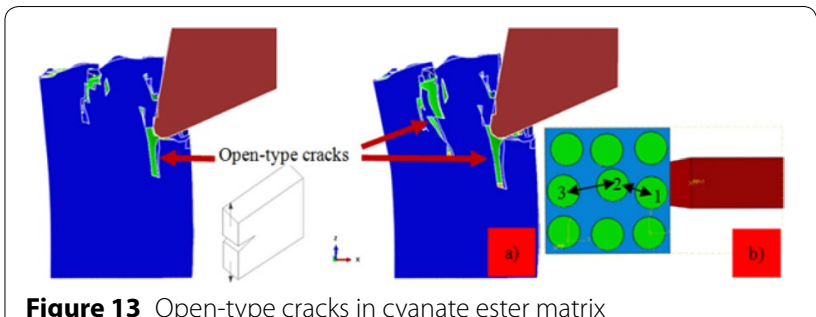

Figure 13 Open-type cracks in cyanate ester matrix

process of each crack type in the carbon fiber/cyanate ester composites is presented.

First, the formation process of open-type cracks in the matrix was evaluated by the simulation of the microcutting process of carbon fiber/cyanate ester composites. As shown in Figure 13, the open-type cracks were easily formed in the matrix. In the composites, the carbon fibers were randomly distributed in the cyanate ester material. Due to the inhomogeneous distribution of carbon fibers in the matrix, the thickness of the matrix material between neighboring carbon fibers varied, thereby resulting in the inhomogeneous mechanical strengths of the matrix materials. In the cutting process, if the thickness of the matrix material between neighboring carbon fibers along the cutting direction is lower, open-type cracks are easily formed. As illustrated in Figure 13(b), it was assumed that three carbon fibers (labelled as 1, 2, and 3) are randomly distributed along the cutting direction. The distance between fibers 1 and 2 was smaller than that between fibers 2 and 3; hence, the mechanical strength of the matrix between fibers 1 and 2 was lower. During the cutting process, under the extrusion of the tool, the stress concentration region is preferentially formed in the matrix with the lower mechanical strength (e.g., the matrix between fibers 1 and 2), thus inducing the further formation of cracks in the matrix. Moreover, the cracks are easily extended along the axial direction of the carbon fibers.

Figure 14 demonstrates the formation process of the tear-type cracks in the matrix. It can be clearly observed 


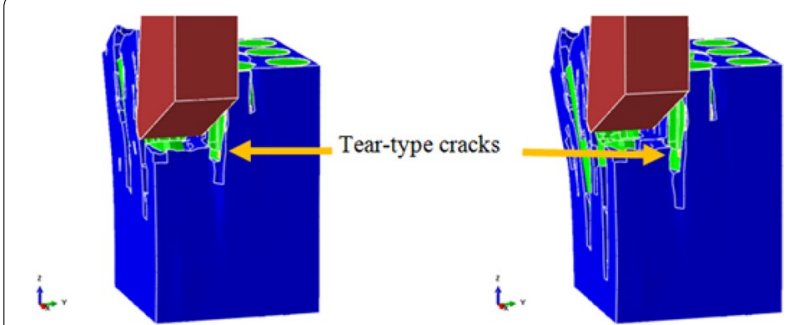

Figure 14 Tear-type cracks in cyanate ester matrix

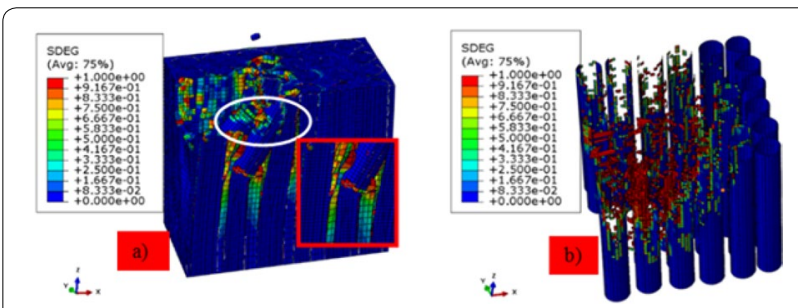

Figure 15 "Open" and "sliding" type cracks in interfacial phase

that tear-type cracks are typically formed in the intersection region between the machined and unmachined regions of the workpiece. Under the extrusion interactions of the tool, the workpiece material is extruded from the workpiece in the cutting direction, thereby leading to the formation and extension of tear-type cracks.

\subsubsection{Machining Damage of Interfacial Phase}

As shown in Figure 15(a), as the micro tool comes into direct contact with the carbon fibers, under the extrusion of the tool, the carbon fibers deform and are damaged. Moreover, in the cutting process, the matrix material that surrounds the carbon fibers tend to be influenced by the uncut part of the matrix material. When the force that acts on the interfacial phase between the carbon fibers and matrix material is increased to a value larger than the ultimate strength of the interfacial phase, cracks could form in the interface. Moreover, these cracks tend to extend along the axial direction of carbon fibers (see Figure 15(b)). In summary, due to the tension and axial forces between the carbon fibers and matrix material, "open" and "sliding" crack types could be formed.

\subsection{Theoretical Analysis for Machining Damage of Carbon Fiber/Cyanate Ester Composites}

Based on the micro geometric model and material model of the carbon fiber/cyanate ester composites, the forces on the single carbon fiber and its surrounding cyanate ester matrix induced by the tool were analyzed, as shown in Figure 16.

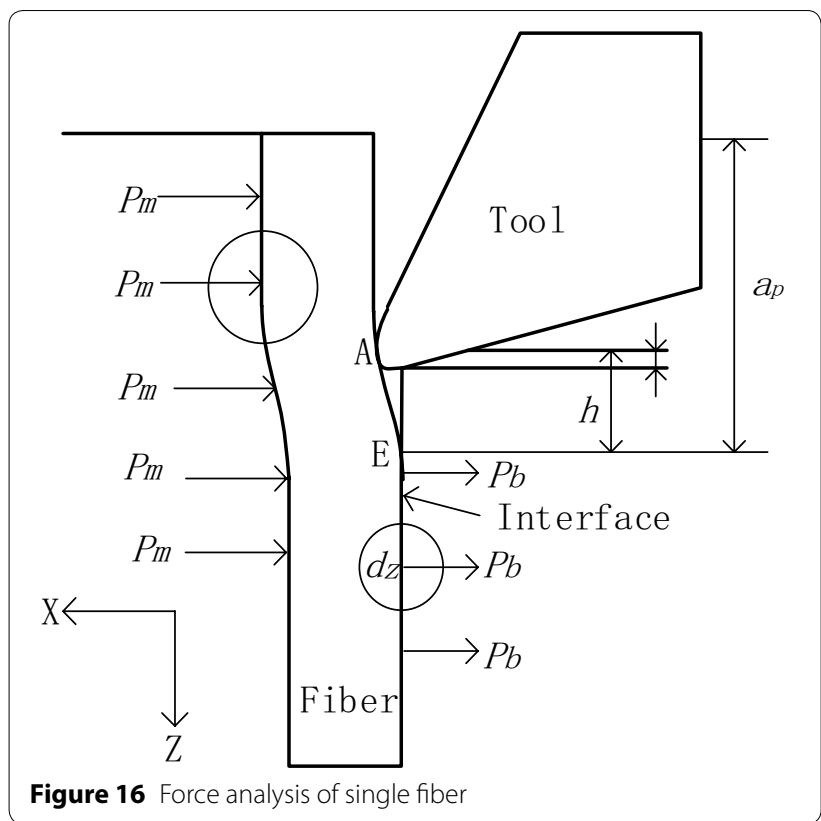

During the cutting process, the fiber is subjected to the shear and squeezing action of the tool, the supporting action of the remaining material, and the bonding action of the interface. To facilitate the analysis, the material used was equivalent to a homogeneous material, with the exception of the carbon fiber, under the assumption that the deflection of a point on the carbon fiber in the $X$ direction is $x$. Hence, the reaction force provided by the remaining support material at this point is

$$
P_{m}=k_{m} x
$$

where $k_{m}$ is the elastic modulus of the equivalent homogeneous material, and it can be calculated using the Biot formula [31], as shown in Eq. (3):

$$
k_{m}=\frac{0.95 E_{m}}{\left(1-v_{m}^{2}\right)}\left[\frac{D^{4} E_{m}}{E_{f} I_{f}\left(1-v_{m}^{2}\right)}\right]^{0.108},
$$

where $E_{m}$ is the transverse Young's modulus of the equivalent homogeneous material (MPa), $v_{m}$ is Poisson's ratio of the equivalent homogeneous material, $E_{f}$ is the Young's modulus of the carbon fiber (MPa), and If is the inertia torque in the transverse section of the carbon fiber $\left(\mathrm{m}^{4}\right)$.

Given that the support material provides the reaction force to the fiber, the cohesive force between the fiber and interface resists the fiber deflection due to the cutting force. When the separation force between the fiber and matrix is higher than the interfacial bond strength, interfacial cracks occur, and they are easily extended in accordance with an increase in the deflection of the carbon fibers. It was 
assumed that the cohesive force at a point in the uncracking interface is

$$
P_{b}=k_{b} x,
$$

where $k_{b}$ is the equivalent elastic modulus of the interface. In this case, the cohesive force at the starting point of the interface cracking is equal to the interfacial bond strength $\sigma_{b}$. Under the consideration of an infinitely small length $d_{z}$ of the carbon fiber, according to the force balance, the balance equation can be expressed as shown in Eq. (5):

$$
\left\{\begin{array}{l}
\sum Q=0, Q-(Q+\mathrm{d} Q)+p_{m} \mathrm{~d} z+p_{b} \mathrm{~d} z=0, \\
\sum M=0, \mathrm{~d} M-(Q+\mathrm{d} Q) \mathrm{d} z-\left(k_{m}+k_{b}\right) \mathrm{d} x \mathrm{~d} z=0,
\end{array}\right.
$$

where $Q$ is the shear force on the fiber $(\mathrm{N})$, and $M$ is the bending moment of the fiber $(\mathrm{N} \cdot \mathrm{m})$. According to the theory of beam bending:

$$
\left\{\begin{array}{l}
Q=-\frac{\mathrm{d}}{\mathrm{d} x}\left(E_{f} I_{f} \frac{\mathrm{d}^{2} x}{\mathrm{~d} z^{2}}\right), \\
M=-E_{f} I_{f} \frac{\mathrm{d}^{2} x}{\mathrm{~d} z^{2}} .
\end{array}\right.
$$

Eq. (6) can be substituted into Eq. (5):

$$
E_{f} I_{f} \frac{\mathrm{d}^{4} x}{\mathrm{~d} z^{4}}+\left(k_{m}+k_{b}\right) x=0 .
$$

Eq. (7) reveals that the deflection curve of the carbon fiber can be solved and divided into three sections: above the point $\mathrm{A}$, between points $\mathrm{A}$ and $\mathrm{E}$, and below point $\mathrm{E}$. Under the assumption that $F_{\text {react }}$ is the reaction force provided by the support material and interface to the single fiber, it could be calculated as follows:

$$
\begin{aligned}
& F_{\text {react }}(x)=\int_{0}^{a_{p}-r_{e}} k_{m} x \mathrm{~d} z+\int_{a_{p}-r_{e}}^{a_{p}+h-\delta} k_{m} x \mathrm{~d} z \\
& +\int_{a_{p}+h-\delta}^{+\infty}\left(k_{m}+k_{b}\right) x \mathrm{~d} z .
\end{aligned}
$$

Hence, the reaction force above point $\mathrm{E}$ can be expressed as

$$
F_{\text {react } 1}(x)=\int_{0}^{a_{p}-r_{e}} k_{m} x \mathrm{~d} z+\int_{a_{p}-r_{e}}^{a_{p}+h-\delta} k_{m} x \mathrm{~d} z,
$$

and the reaction force below point $\mathrm{E}$ is

$$
F_{\text {react } 2}(x)=\int_{a_{p}+h-\delta}^{+\infty}\left(k_{m}+k_{b}\right) x \mathrm{~d} z .
$$

Moreover, the fibers are subjected to squeezing and shearing by the tool nose at the contact point. The deformation failure of CFRP composites during the cutting process could be summarized as follows.

When the fibers have no initial defects, under the supposition that the support force of the foundation material and the bond strength of the interface are significant, the fiber and the matrix do not crack, and the fiber is cut off directly by the tool. Therefore, no micro cracks occur on the machined surface. Conversely, if the support force of the foundation material and the bond strength of the interface are very small, the interface cracks when the cohesive force provided by the interface reaches the cohesive strength. The extrusion force produced by the fiber on the foundation material increases until the matrix is crushed. The machined surface of the composites therefore contain defects due to the interface cracking and matrix crushing.

When the fibers have clear initial defects, the strength of a single fiber is randomly distributed, and the weakest part of each fiber is exposed. If the maximum normal stress in the fiber is greater than the strength limit, the fiber fractures. Otherwise, the fiber continues to bend until it is either cut off or fractures. When the location of the initial defect is close to the cutting surface, the fracture depth of the carbon fiber is shallow, or the fiber is easy to fracture at a greater depth. The fracture of a single carbon fiber can induce hole defects, and the location of the fiber breakage has a direct influence on the depth of the defects.

\section{Conclusions}

In this study, a three-phase micro model that consists of carbon fiber, cyanate ester, and an interface phase was established. The periodic random distribution of the carbon fiber reinforced phase in the matrix was realized using the double perturbation algorithm. To achieve the stochastic distribution of the single carbon fiber strength, a novel method based on the Weibull intensity distribution theory and Monte Carlo method was proposed. The model can accurately simulate the microstructure and mechanical properties of the composites. Moreover, the damage mechanism of the composites during the cutting process was evaluated. The main conclusions can be summarized as follows:

1. The processed surfaces of the carbon fiber/cyanate ester composites were observed using SEM. The experimental results were approximately consistent with the simulation results, which verifies the simulation model. The damage on the machined surface mainly includes hole defects caused by the fracture of local single carbon fibers, surface cracks due to the elastic bending deformation of carbon fibers, and cavities or incomplete adhesion between the carbon 
fibers and matrix. This is in addition to the elongated cracks induced by the shedding of the fibers.

2. The mechanism of the machining damage of threephase composites during the cutting process was analyzed using finite element simulations. There are two types of failures that occur on carbon fibers during processing: shearing fracture and bending fracture. Open-type or tear-type cracks occur in the cyanate ester matrix, and the deformation of the fibers can also lead to matrix crushing. In addition, the interface mainly contains open-type and sliding-type cracks.

3. The theoretical analysis model on the microscopic damage of carbon fiber/cyanate ester composites is presented, and the cutting resistance force between the matrix and single fiber interface was derived. Furthermore, the deformation failure of the CFRP composites during the cutting progress is summarized for the two cases, both of which could result in interface cracking and matrix damage. When the initial defects are present on fibers, the fibers tend to bend and fracture, which can induce hole defects. Otherwise, the fibers are directly cut off by the tool.

\section{Authors' Contributions}

HL was in charge of the whole trial; $J L$ and YS wrote the manuscript; JZ assisted with the sampling and laboratory analyses. All authors read and approved the final manuscript.

\section{Author Details \\ ${ }^{1}$ Center for Precision Engineering, School of Mechanical Engineering, Harbin Institute of Technology, Harbin 150001, China. ${ }^{2}$ Rainbow Drone Technology Co., Ltd, China Academy of Aerospace Aerodynamics, Beijing 100000, China.}

\section{Authors' Information}

Haitao Liu, born in 1982, is currently an associate professor at Center for Precision Engineering, Harbin Institute of Technology, China. He received his PhD degree from Harbin Institute of Technology, China, in 2010. His research interests include ultra-precision machine and and machining technology.

Jie Lin, born in 1995, is currently a master candidate at State Key Laboratory of Precision and Ultra-precision Machining, Harbin Institute of Technology, China.

Yazhou Sun, born in 1968, is currently a professor at Center for Precision Engineering, Harbin Institute of Technology, China. He received his PhD degree from Harbin Institute of Technology, China, in 2005. His research interests include ultra-precision machine and machining technology.

Jinyang Zhang, born in 1992, is currently a mechanical engineer at Rainbow Drone Technology Co., Ltd, China Academy of Aerospace Aerodynamics, China. He received his master degree from Harbin Institute of Technology, China, in 2017.

\section{Competing interests}

The authors declare that they have no competing interests.

\section{Funding}

Supported by Research Innovation Fund Project "Research on micro machining mechanism of fiber reinforced composites" (Grant No. HIT. NSRIF.2014055) of Harbin Institute of Technology, China.

Received: 13 July 2018 Accepted: 22 May 2019

Published online: 10 June 2019

\section{References}

[1] B N Nguyen, M A Khaleel. A mechanistic approach to damage in shortfiber composites based on micromechanical and continuum damage mechanics descriptions. Composites Science and Technology, 2004, 64(5): 607-617.

[2] N Feito, J López-Puente, C Santiuste, et al. Numerical prediction of delamination in CFRP drilling. Composite Structures, 2014, 108(1): 677-683.

[3] MYWu, Y Gao, Y N Cheng, et al. Carbon fiber composite materials finite element simulation analysis of cutting force. Procedia Cirp, 2016, 56: 109-114.

[4] S Sugita, H Awaki, K Yoshioka, et al. Studies of print-through and reflectivity of $\mathrm{x}$-ray mirrors using thin carbon-fiber-reinforced plastic. Journal of Astronomical Telescopes Instruments \& Systems, 2016, 2(1): 014002.

[5] P Kavouras, D A Dragatogiannis, D I Batsouli, et al. Effect of local microstructure on the indentation induced damage of a fiber reinforced composite. Polymer Testing, 2017, 61: 197-204.

[6] S Sakata, F Ashida, K Enya. A microscopic failure probability analysis of a unidirectional fiber reinforced composite material via a multiscale stochastic stress analysis for a microscopic random variation of an elastic property. Computational Materials Science, 2012, 62: 35-46.

[7] M R Abir, TE Tay, M Ridha, et al. Modelling damage growth in composites subjected to impact and compression after impact. Composite Structures, 2017, 168: 13-25

[8] RW Hillermeirer, J C Seferis. Environmental effects on thermoplastic and elastomer toughened cyanate ester composite systems. Journal of Applied Polymer Science, 1997, 77(3): 556-567.

[9] X M Wang, L C Zhang. An experimental investigation into the orthogonal cutting of unidirectional fibre reinforced plastics. International Journal of Machine Tools \& Manufacture, 2003, 43(10): 1015-1022.

[10] D N Bhatnagar, D Nayak, I Singh, et al. Determination of machininginduced damage characteristics of fiber reinforced plastic composite laminates. Advanced Manufacturing Processes, 2004, 19(6): 1009-1023.

[11] N Shetty, S M Shahabaz, S S Sharma, et al. A review on finite element method for machining of composite materials. Composite Structures, 2017, 176: 790-802.

[12] Y Su, Z Jia, B Niu, et al. Size effect of depth of cut on chip formation mechanism in machining of CFRP. Composite Structures, 2017, 164: 316-327.

[13] A Mkaddem, M E Mansori. Finite element analysis when machining UGFreinforced PMCs plates: Chip formation, crack propagation and induceddamage. Materials \& Design, 2009, 30(8): 3295-3302.

[14] M Mahdi, L Zhang. An adaptive three-dimensional finite element algorithm for the orthogonal cutting of composite materials. Journal of Materials Processing Technology, 2001, 113(1): 368-372.

[15] M Mahdi, L Zhang. A finite element model for the orthogonal cutting of fiber-reinforced composite materials. Journal of Materials Processing Technology, 2001, 113(1): 373-377.

[16] X C Sun, M R Wisnom, S R Hallett. Interaction of inter- and intralaminar damage in scaled quasi-static indentation tests: Part 2 - Numerical simulation. Composite Structures, 2016, 136: 727-742.

[17] E Abisset, F Daghia, X C Sun, et al. Interaction of inter- and intralaminar damage in scaled quasi-static indentation tests: Part 1 - Experiments. Composite Structures, 2016, 136: 727-742.

[18] GV G Rao, P Mahajan, N Bhatnagar. Three-dimensional macro-mechanical finite element model for machining of unidirectional-fiber reinforced polymer composites. Materials Science and Engineering A, 2008, 498: 142-149.

[19] S Zenia, L B Ayed, M Nouari, et al. Numerical prediction of the chip formation process and induced damage during the machining of carbon/ epoxy composites. International Journal of Mechanical Sciences, 2015, 90: 89-101.

[20] S Zenia, L B Ayed, M Nouari, et al. Numerical analysis of the interaction between the cutting forces, induced cutting damage, and machining parameters of CFRP composites. The International Journal of Advanced Manufacturing Technology, 2015, 78(1-4): 465-480.

[21] A Dikshit, J Samuel, R E Devor, et al. A microstructure-level material model for simulating the machining of carbon nanotube reinforced polymer composites. Journal of Manufacturing Science and Engineering, 2008, 130: 031110.

[22] R Rentsch, O Pecat, E Brinksmeier. Macro and micro process modeling of the cutting of carbon, Procedia Engineering, 2011, 10: 1823-1828. 
[23] A Abena, S L Soo, K Essa. A finite element simulation for orthogonal cutting of UD-CFRP incorporating a novel fibre-matrix interface model. Procedia CIRP, 2015, 31: 539-544.

[24] F J Wang, X N Wang, R Yang, et al. Research on the carbon fibre-reinforced plastic (CFRP) cutting mechanism using macroscopic and microscopic numerical simulations. Journal of Reinforced Plastics and Composites, 2017, 36(8): 555-562.

[25] L Yang, Z Wu, D Gao, et al. Microscopic damage mechanisms of fibre reinforced composite laminates subjected to low velocity impact. Computational Materials Science, 2016, 111: 148-156.

[26] J H Lu, P Zhu, Q H Ji, et al. Identification of the mechanical properties of the carbon fiber and the interphase region based on computational micromechanics and Kriging metamodel. Computational Materials Science, 2014, 95: 172-180.

[27] M Yu, P Zhu, Y G Ma. Identification of the interface properties of hollow spheres filled syntactic foams: An inverse strategy combining microstructural modeling with Kriging metamodel. Composites Science and Technology, 2013, 74: 179-185.
[28] T Okabe, N Takeda. Estimation of strength distribution for a fiber embedded in a single-fiber composite: experiments and statistical simulation based on the elasto-plastic shear-lag approach. Composites Science and Technology, 2001, 61(12): 1789-1800.

[29] K Naito, J M Yang, Y Tanaka, et al. The effect of gauge length on tensile strength and Weibull modulus of polyacrylonitrile (PAN)-and pitch-based carbon fibers. Journal of Materials Science, 2012, 47(2): 632-642.

[30] J Che, M B Chan-Park. Reactive spinning of cyanate ester fibers reinforced with aligned amino-functionalized single wall carbon nanotubes. Advanced Functional Materials, 2008, 18(6): 888-897.

[31] W Xu, L C Zhang. On the mechanics and material removal mechanisms of vibration-assisted cutting of unidirectional fibre-reinforced polymer composites. International Journal of Machine Tools and Manufacture, 2014, 80: 1-10.

\section{Submit your manuscript to a SpringerOpen ${ }^{\circ}$ journal and benefit from:}

- Convenient online submission

- Rigorous peer review

- Open access: articles freely available online

- High visibility within the field

- Retaining the copyright to your article

Submit your next manuscript at $\boldsymbol{\nabla}$ springeropen.com 